\title{
UAV-Assisted Hybrid Scheme for Urban Road Safety Based on VANETs
}

\author{
Sayed Jobaer@, Yihong Zhang *, Muhammad Ather Iqbal Hussain $₫$ and Foysal Ahmed
}

College of Information Science and Technology, Engineering Research Center of Digitized Textile \& Fashion Technology, Ministry of Education, Donghua University, Shanghai 201620, China; 318058@mail.dhu.edu.cn (S.J.); 415030@mail.dhu.edu.cn (M.A.I.H.); 319030@mail.dhu.edu.cn (F.A.)

* Correspondence: zhangyh@dhu.edu.cn; Tel.: +86-138-1782-6259

Received: 15 August 2020; Accepted: 7 September 2020; Published: 12 September 2020

\begin{abstract}
Traffic congestion control is becoming a popular field of research due to the rapid development of the automotive market. Vehicular ad hoc networks (VANETs) have become the core research technology for numerous application possibilities related to road safety. Road congestions have been a serious issue of all time since the nodes have high mobility and transmission range is limited, resulting in an interruption of communication. One of the significant technical challenges faced in implementing VANET is the design of the routing protocol, providing adequate information and a reliable source for the destination. We proposed a novel mechanism unmanned aerial vehicle (UAV)-assisted ad hoc on-demand distance vector (AODV) routing protocol (UAVa) for current-time traffic information accumulation and sharing to the entire traffic network and to control congestions before it happens. The UAV-assisted (UAVa) protocol is dedicated to urban environments, and its primary goal is to enhance the performance of routing protocols based on intersections. We compared the overall performance of existing routing protocols, namely ad hoc on-demand distance vector. The simulations were done by using OpenStreetMap (OSM), Network Simulator (NS-2.35), Simulation of Urban Mobility (SUMO), and VanetMobiSim. Furthermore, we compared the simulation results with AODV, and it shows that UAV-assisted (UAVa) AODV has significantly enhanced the packet delivery ratio, reduced the end-to-end delay, improved the average and instant throughput, and saved more energy. The results show that the UAVa is more robust and effective and we can conclude that UAVa is more suitable for VANETs.
\end{abstract}

Keywords: VANET; UAV; AODV; NS-2; SUMO; congestion control

\section{Introduction}

Vehicular ad hoc networks (VANETs) is an application of mobile ad hoc networks (MANETs) for the field of the internet of vehicles (IoV) [1,2]. Compared with other mobile ad hoc networks, VANET is a network that can communicate wirelessly among the vehicles, and between the roadside units (RSUs) to vehicles [3]. Intelligent Transportation System (ITS) applications are being supported by VANETs. It is designed for the comfort and safety of the passengers and also to improve the transportation efficiency [4]. For the new application scenarios, inter-vehicle communication (IVC) has aroused widespread research interest in academia as well as industry [5]. The vehicles are treated as autonomous mobile nodes, connected in an environment wirelessly, though these nodes are not present in similar range, passing through multiple intermediate nodes [6]. The VANETs build and maintain ad hoc networks with the aid of routing protocols [7]. The routing protocol proposed in VANETs may be table-driven (proactive), on-demand-driven (reactive), or hybrid $[8,9]$. The on-demand (reactive) routing protocols discussed here are ad hoc on-demand distance vector (AODV) and our proposed unmanned aerial vehicle (UAV)-assisted AODV (UAVa). For mobile ad hoc networks a routing is 
important [10]. A lot of work has been conducted into routing protocols [11]. Many protocols are aimed at delivering higher quality of service (QoS) and saving more resources [12]. AODV is the most popular routing protocol which contains the technique of route discovery and route maintenance [13]. The source node transmits the packet of the routing requests to the network destination node. After the routing request packet has been received, the intermediate node must forward the packet before the routing request is received by the destination node, and the repeat request packet is discarded [14]. The AODV routing protocol also attaches the sequence number to the request packet to control the routing loops at the same time. Without any transmission requirements, the proactive routing protocol can create routes between nodes in advance. This is different from the reactive routing protocol, i.e., a node only updates its neighbors so far with its known routing information, assuming that all nodes will use the whole network updated to the information [15]. Private vehicles have become an integral part of our everyday lives because of the rapid growth of our society, the rise in individual vehicles; traffic congestion has become an acute issue that needs to be addressed [16]. In our proposed scheme, congestion control is our first target. Nowadays, congestion regulation has become a widely debated concern in vehicle-to-vehicle (V2V) communications, i.e., ensuring secure and effective communications through the use of limited resources available in vehicular ad hoc networks (VANETs) [17]. A node in VANET represents the vehicle.

The shortcomings of existing congestion control mechanisms over VANETs have inspired us to propose an approach with an improvement in the existing algorithms to meet the demands of current technology in handling vehicles in addition to assuring safety in communicating vehicles with reliability. The issues faced before introducing the solutions to the congestion in VANETs were limited bandwidth, high mobility, retention of connection amongst the moving nodes, avoidance of interference and noise, maintaining signal strength in the presence of the dense building on the sides of the roads, etc. [18].

In this article, an innovative mechanism is proposed in which current-time traffic information accumulation and sharing to the entire traffic network and how to regulate congestions before it happens are studied. This not only ensures the sharing efficiency but also demonstrates the low complexity of accumulating the current-time traffic information and sharing it. Within the existing conditions of traffic, the traffic rules were completely used to remove any congestion. We performed a simulation-based performance assessment of our UAVa protocols and demonstrated the efficacy and robustness of UAVa in terms of adding routing efficiency, average throughput, instant throughput, reduced latency, and improved delivery ratio and residual energy compared to AODV protocols. The processes and algorithms will be further discussed in the following sections.

The rest of this paper is structured as follows: In Section 2, we present examples and discussions of intersection-based congestion control routing protocols. In addition to the details of the proposed UAVa routing protocol and algorithms, key estimates and features are provided in Section 3, the simulation and evaluation processes are described in Section 4; and the conclusions are finally drawn in Section 5.

\section{Related Works}

For controlling congestion, current-time information sharing is very important. This becomes a significant challenge for sharing current-time information in VANETs due to the imbalance of traffic flow on the road network and sudden obstructions, blockages, or traffic accidents. To achieve a mechanism for the current-time sharing of information in a fast-moving node transportation network, a road-based vehicular traffic (RBVT) routing through VANETs presented in [19]. In [6], the authors present a method for estimating travel time from a source place in a road network to a destination place called Travel Time Assessment (TTE), which serves as a route planning metric. They proposed a current-time route planning algorithm based on TTE comparisons by collecting data to avoid traffic congestion in an intersection. In [20], to improve the connectivity of intersection-based routing, the authors have introduced the connectivity of junction-based routing (CJBR). Like other routing 
protocols focused on intersections, CJBR disregards dependence on traffic density metrics for the selection process of intersections during the development of routing paths in urban environments.

The author has designed a flooding model in [21] that automatically responds to any change in topology while overcoming current obstacles. At the same time, in an ad hoc fashion, exchange data with a drone, commonly known as an unmanned aerial vehicle (UAV) in this work, explores well-regulated routing paths that can provide long-life connectivity. UAV's assistance to vehicles will provide a high transfer rate and low transfer latency, while effectively extending the network link, depending on the amount of traffic and the end time of each discovered path. In Silva et al. [22] other functions are introduced to enhance the efficiency of its proposed algorithm, including (1) Using the data in the extended neighboring table for optimal route selection, and seek to prevent neighbors from reaching local maximums on each destination route and avoiding the loop of data packets. (2) Substitute the constant greedy forwarding with the right-hand rule in recovery mode, and the left-hand rule fused at the same time. Guo et al. [23] proposed an optimization model, which reduces the amount of total travel time on the road network and the time representation of the system's impact of traffic imbalances. The author introduces the analytical expression of the problem of optimization and proposes an algorithm, the dynamic interior point method (DIPM), which by rerouting the driver solves this problem of optimization.

In [24], the author proposes an optimization model that not only increases traffic flow but also avoids traffic congestion to improve road utilization. The algorithm uses a real map for simulating the area, with a large amount of traffic data. In [25], the author provides an analytical framework to study UAV-to-UAV communication underlay with the uplink of a cellular network. In their work, they proposed a realistic channel model, antenna pattern, and power control strategy equation obtained. In [26], the author proposed a protocol named early congestion detection and control routing protocol (EDAODV). This protocol is applicable to one-way highways and contrasted to AODV protocol because it is non-adaptive to routing congestion in VANETs.

However, previous congestion control methods can not resolve the effects of the present traffic conditions, such as the traffic flow on-road segments and the waiting queue at the intersections. Based on the UAV-assisted traffic congestion control method, there are several technologies and algorithms based on intersection congestion control methods in VANETs. In [27], the author proposed the UAV-assisted VANETs routing protocol (UVAR) and its extensions to support self-organized routing between VANETs and UAVs as well as UAVs themselves. The UVAR protocol is designed for urban scenarios, and its primary goal is to enhance the routing efficiency based on UAV's knowledge of ground road traffic. Moreover, the combination of RSUs and UAVs at the intersection for controlling congestion is remaining.

\section{UAV Assisted (UAVa) Reactive Protocol Strategy}

After describing some of the proposed work in the literature and mentioning their shortcomings, this section will describe UAVa and its key processes.

1. Route Discovery Process: The route discovery scheme is used to discover the suitable routes between communication networks, the selected route may include all types of equipment used as relays (e.g., Vehicles, RSUs, or UAVs).

2. Route Selection Process: The source node analyzes various metrics and selects the shortest route from the source to the destination using Dijkstra's famous greedy algorithm.

3. Packet Delivery Process: Data packets are converted along their respective routing paths.

4. Replan and Revise Process: Due to the dynamic topology of the network, a permanent connection to the selected routing route cannot be achieved. Therefore, always looking for alternative routes is an essential condition. 
Before unfolding the effectiveness of our proposed scheme, a preliminary description of the communication method between nodes and our proposed UAVa system model is required. Later, the key process of our proposed UAVa protocol will be described in detail.

\subsection{UAVa's System Model}

UAVa is proposed to reduce the dependence on the traffic density for reducing the congestion and intermediate intersections selection between the source node and the destination node, and increase data packet transmission rate, thereby enhancing intersection-based routing mobility by sending data at any time and anywhere irrespective of traffic density. The novelty of this selection mechanism is that the roadside units (RSUs) and unmanned aerial vehicles (UAVs) at road intersections are among the selection metrics, where RSUs are very important in VANETs. Yet, RSUs were not previously used as metric-assisted UAVs for intersection.

Installing RSUs every $200 \mathrm{~m}$ is a lot of capital cost. Reducing the capital cost of deploying RSU is justified by adding a limited number of RSUs to create the intersections. As an independent network, it is assumed that RSUs communicate with each other through the existing cellular network, i.e., there is no direct wireless or wired contact from RSU to RSU. UAVa assumes a vast and continuous traffic density at a certain period of time cycle, and that each node can download a digital map through the vehicle to roadside $(\mathrm{V} 2 \mathrm{R})$ communications.

Let us illustrate the different assumptions considered by the system model of UAVa. Consider VANETs stationed in a geographically small urban area where a group of RSUs is implemented on the ground, and UAVs are hovering in the congested intersection. Both RSUs and UAVs communicate with vehicles on the ground and exchange data. As shown in Figure 1, the road intersection is RSU based. UAVs will be assisted up to its demands. Assume that it is divided into fixed intersections with an ID. The communication range of these intersections is defined based on the RSUs capacity. For understanding our proposed network, different static connections are characterized by a unique ID, since our routing process is not primarily based on static nodes, but rather depends on the intersection. This research is essential for understanding relevant information, such as the present traffic conditions, the uniform allocation of the RSUs, and the destination location. The geographical positions and the identity of every intersection should be known by all nodes and UAVs utilizing their downloaded digital city map through V2R communications. Based on the embedded on-board unit (OBU) with GPS, every node can determine its current geographical location. In addition, we assume that there is a limitation of vitality for all nodes since they can be equipped with rechargeable batteries at first. In our proposed scheme, each node energy is 300 joules.

It should be emphasized that UAVs float very well at low altitudes to operate on the ground vehicles. We assume that RSUs are allocated uniformly across the network and moving vehicles which do not exceed $300 \mathrm{~m}$ during the flight of UAVs. Through following cellular standards, RSUs can communicate with each other on a wireless interface. As depicted in Figure 1, we use six forms of wireless configuration to build the entire network communications we propose:

(1) Vehicle-to-Vehicle (V2V): Vehicles on the ground can communicate according to the IEEE 802.11p wireless standard within the line-of-sight (LoS) communication range with each other. In the event of an obstacle, communication is not possible.

(2) Vehicle-to-Unmanned aerial vehicle (V2U): Vehicles and UAVs can communicate with each other according to the IEEE 802.11p wireless standard. In general, UAVs in urban areas do not fly at high altitudes, because majority of their applications in our chosen simulation scenario must be carried out at low altitudes.

(3) Vehicle-to-Roadside unit (V2R): For most instances, this form of contact exists for applications requiring direct access to the information provided by the gateways (i.e., RSUs). Vehicle and RSU at a road intersection can communicate with each other based on the inclusion of a wireless protocol that adopts the IEEE 802.11p standard, which can have a high likelihood of line-of-sight (LoS). 
(4) Cellular network-to-Road side unit (C2R): We consider the uplink of a traditional ground cellular network, as depicted in Figure 1, where cellular networks are uniformly distributed, and all the cellular networks are deployed at the same height and communicate with their respective sets of connected RSUs. Assuming that the number of RSUs is sufficiently large compared to that of the cellular network, we further consider that RSUs associate to their closest cellular tower, which generally also provides the most significant reference signal received power. Where the 2-D distance between the interfering RSU and the typical cellular network is in a fixed range. Through existing cellular technology, the roadside unit can exchange data between them.

(5) Unmanned aerial vehicle-to-Road side unit (U2R): UAV-based applications need their proper functionality through internet access. We proposed the IEEE 802.11p standard for UAV to RSU communication. UAV connects to an RSU within range, either to deliver their current applications or to relay packets of data.

(6) Cellular-to-Cellular (C2C): Cellular technology will share data between them if the distances between them are longer than the ranges of each.

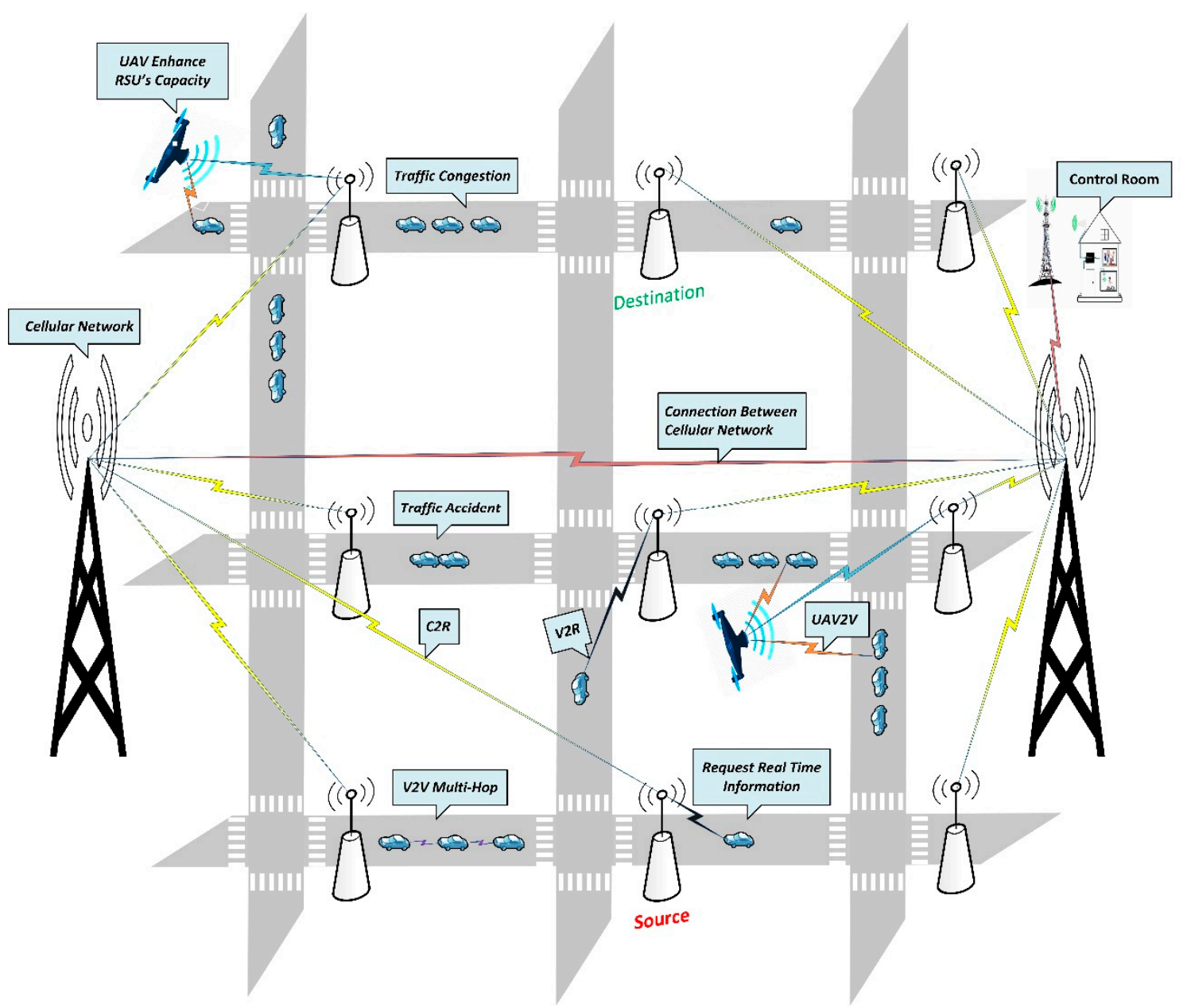

Figure 1. Unmanned aerial vehicle (UAV)-assisted hybrid scheme for urban road safety based on the vehicular ad hoc network (VANET) model.

\subsection{UAVa's Design Approach}

To introduce the current-time congestion control process, we propose the network architecture, and then define the definition and underlying assumptions of the road network. Our proposed UAVa protocol is similar to other routing protocols based on intersections. The UAVa protocol represents six 
routing types, i.e., routing with $\mathrm{V} 2 \mathrm{~V}$ in road segments, routing at road intersections with $\mathrm{V} 2 \mathrm{R}, \mathrm{V} 2 \mathrm{U}$, $\mathrm{U} 2 \mathrm{R}, \mathrm{C} 2 \mathrm{R}$, and routing between existing C2C cellular networks.

\subsubsection{UAVa's Network Architecture}

UAV's network architecture is shown in Figure 2. Here we represent different kinds of scenarios, road segment nodes, and road intersection allocated RSUs. In an emergency basis, such as sudden congestions or blockage, UAVs assist in RSU at road intersections to increase the RSU's coverage and capacity to regulate the situation. We consider that every node is equipped with an on-board unit (OBU), which aims to achieve V2V communication with other nodes on the road segments and V2R and V2U connection with RSU and UAV in the road intersections. OBU records overall information of a node, such as nodes ID, position, current-time speed, etc. It obtains details on the subsequent road intersection from the RSU through V2R communications. The source node can calculate the travel time based on the current-time speed of the road segment and record it by its OBU. If any blockage occurs on a particular road intersection, such as sudden traffic accidents or congestion, OBU will produce a warning message and send it to RSU, and RSU will relay this warning message through the entire traffic network, which contains the ID of the intersection and the approximated time to release the congestion.

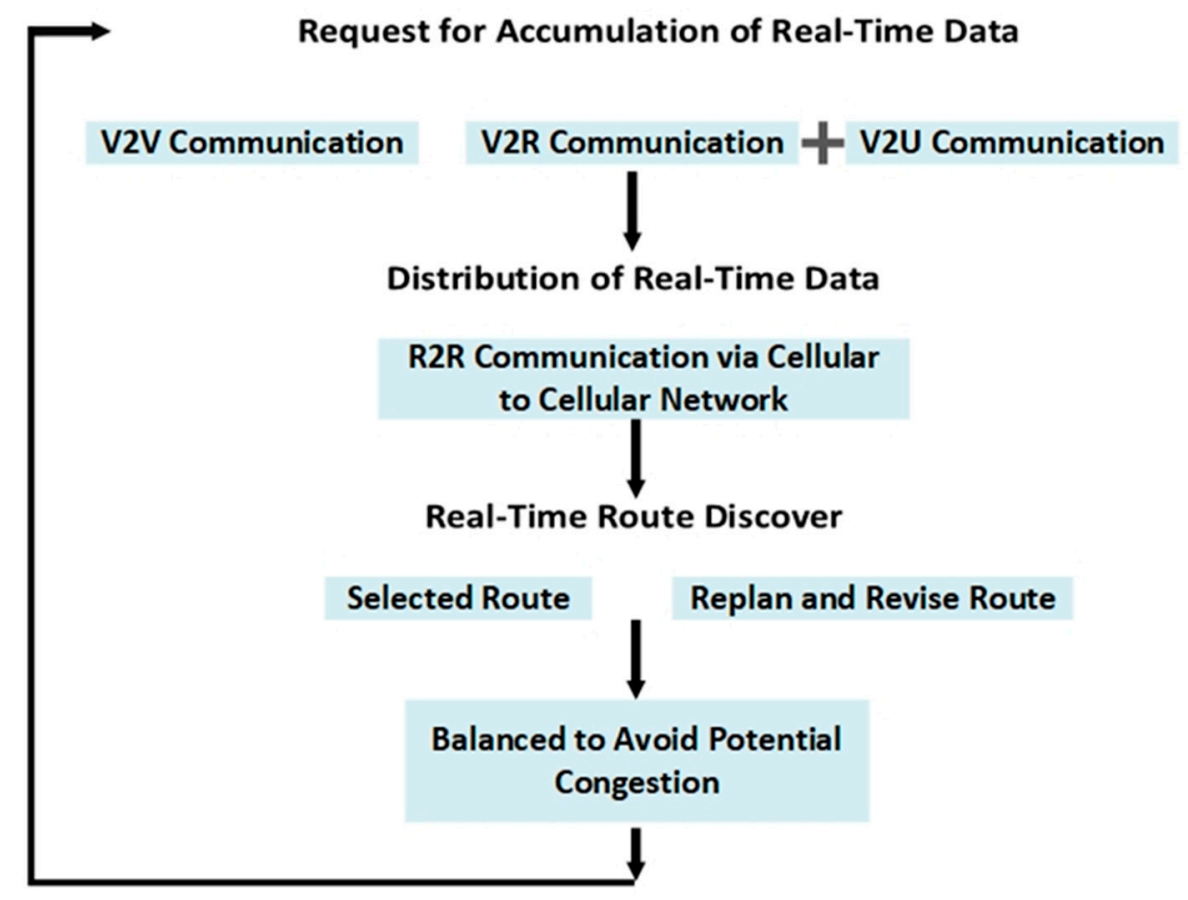

Figure 2. Transportation network architecture.

In VANETs, V2V communication efficiency will be influenced by the speed of linked nodes in the road segments. Different V2V communication mechanisms are used to collect and exchange current-time traffic information according to different requirements of all the connected nodes in the road network. Suppose a large number of linked nodes are installed on the road network, then the current-time traffic information can be exchanged via V2V communication with multi-hop services. Due to the huge amount of connected nodes in the road segments, the source node can find reliable relay nodes within its wireless communication range, and one hop can send current-time traffic data to the relay node. Current traffic data can be transmitted along with the road segments until at the intersection the relay node joins the wireless communication range of the RSU. Using V2R or V2U communications, the RSU or UAV at the intersection may collect current-time traffic information while 
the large majority of connected nodes on the route, the effect of data transmission delay on travel time estimation accuracy, are typically ignored.

When a small part of connected nodes is deployed in a vehicular ad hoc network, current-time traffic information on the road intersections can be accumulated and shared through V2R communication. Due to the low rate of connected nodes, the current-time traffic information accumulated and shared along with the road segments, via the carry-and-forward mechanism, suppose a source node within its wireless communication range cannot find a reliable relay node to share the current-time traffic information. In this scenario, the node will continue to move along the path with current-time traffic information. If a stable relay node is located by the source node, the information will be transmitted by continuing to switch between forward and backward until the relay node reaches the contact range of the RSU at the intersection. The RSU uniformly deployed each intersection to accumulate the current-time traffic information from the mobile nodes through V2R or V2U communications in VATENTs.

In the entire network, the RSUs of the road network are connected through a cellular network, and the R2R communication is realized through our designed current-time traffic data accumulation and sharing mechanism. We considered two types of the traffic data being stored in the memory of RSU, which is the static data type and the dynamic data type. Static data contain the distance of each road segment, road intersection information, the capability of different areas, all of which are obtained through digital maps. Another is dynamic current-time information accumulated from nodes through VANETs or transportation infrastructure, such as traffic surveillance cameras and detection circuits. Connected nodes upload dynamic information via V2R communication or V2U communication, such as sudden traffic congestion caused by unexpected accidents. At the intersection, the traffic monitoring camera and detection circuits track and record real-time traffic information, which includes road density and number of nodes, and then upload the real traffic information to the RSUs at the intersections. Complex traffic details are automatically updated on the entire road network.

In our proposed UAVa's network architecture, RSUs at intersections cover a limited communication area, inside this coverage area the RSU receives the node's request and response, the node arrives at this coverage area and requests to RSU for current-time traffic information through VANETs. RSU shares current traffic information through our designed current-time traffic information accumulation and sharing mechanism, designed to accumulate and share current-time traffic information across the entire road network. The node calculates the travel time estimate and selects the shortest route from the source to the destination through Dijkstra's famous greedy shortest path planning algorithm. Once sudden congestion occurs, immediately current-time traffic information is updated to the entire network. Then the control room will take immediate action, and they launch a UAV to this congested intersection. The UAV will assist RSU in this congested intersection to enhance the coverage area of this intersection, and create a backpressure strategy to avoid new congestion and fixed-route interruption among the source to the destination node. By using the routing table, the source node can modify or revise the route again, and the source node can select the second shortest route for the destination. Our proposed UAVa algorithm is briefly described in Algorithm 1. 


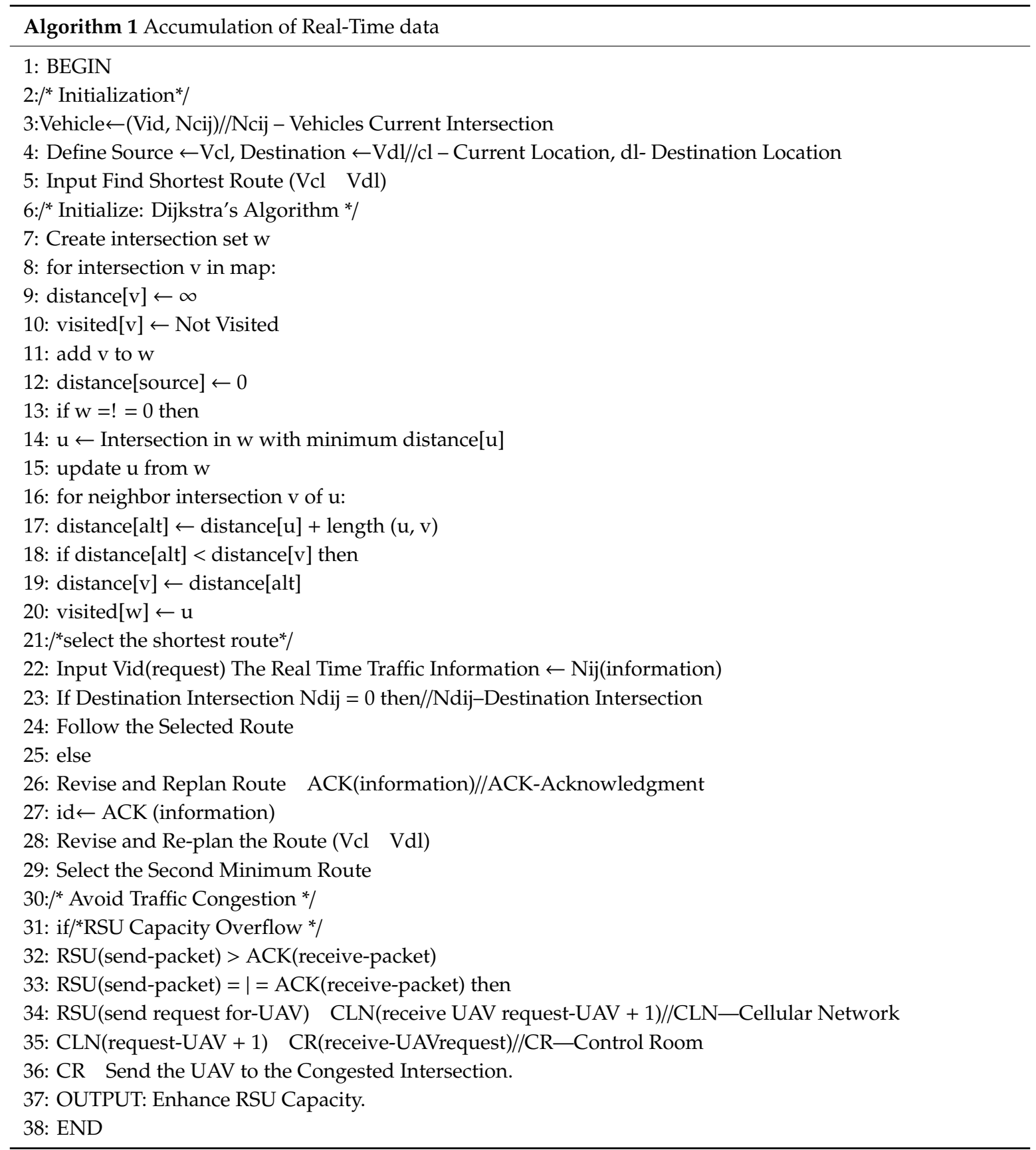

\subsubsection{Route Discover Metrics}

In this section, some of the metrics used in the UAVa routing mechanism are discussed in detail.

Accumulate and Distribute Real-Time Data: We propose an innovative idea that can accumulate and distribute current-time data as well as take immediate steps to prevent congestion at the road intersections. This section will describe our proposed mechanism for current-time information-sharing between nodes to RSUs and UAVs at intersections. The analysis of complexity and intensity demonstrates that the proposed current-time traffic data accumulation and sharing mechanism can transfer information in current-time to the entire network between RSUs, UAVs, and vehicles. We have already mentioned that traffic information stored in RSU memory is divided into two classes. One is static data, containing all information about traffic networks, and previous congestion history; nodes can download static information through V2R communications and reduce measurement errors. Static traffic data are updated between RSUs after a definite period of time. For dynamic traffic data, 
such as unexpected traffic collisions or congestions, RSU's current-time information accumulation and sharing mechanism will immediately transmit these dynamic current-time traffic data over the traffic network instead of periodically updating them. For our proposed model, the current-time traffic information-sharing mechanism for RSU's is broadcast. The broadcasting technique for information sharing has excellent performance. Moreover, our proposed current-time traffic information-sharing mechanism encompasses the entire transmitted traffic network, which likely to be useful for other nodes on the road segments.

RSU Setup Process: In our proposed system, all RSUs are connected via existing cellular networks because of reducing RSU setup costs. There is no need to create a new network setup for RSUs. We are using a carry and forward mechanism in our model to reduce the cost of creating a new RSU network. We proposed to place RSUs at an intersection. One intersection to another intersection distance could be $5 \mathrm{~km}$ to $10 \mathrm{~km}$, and make a wired or wireless connection between RSU to RSU quite impossible because urban area obstacles are so high. To remove those redundancies, we proposed all of our RSUs be connected with the existing cellular network. We assume that all RSUs and UAVs are equipped with a single omnidirectional antenna with unitary gain. Nowadays, cellular technology is well developed and $5 \mathrm{G}$ systems are in our hands. We proposed a different and unique idea for avoiding congestion before it occurs. It is known that issue prevention is better than regulation.

Route Discover and Selection Process: To discover the shortest path from the source to the destination, at first from the source, all the directly connected route distances need to be determined using a digital city map. These distances are the shortest curved metric distance from the source to the destination, where a curve metric distance is a distance determined by using Dijkstra's famous greedy algorithm based on the geometric shape of the road segments by.

As shown in Figure 3, there are several road segments and road intersections, assume source intersection (IBZ1) and the destination intersection (IBX1). Hence, the shortest curve metric distance of IBZ1 means selecting the shortest route of these road segments in terms of distance. Using Dijkstra's famous greedy algorithm, we can choose the shortest route from source intersection to destination intersection. According to this Dijkstra's algorithm, we can calculate the shortest route in our designed model after applying the shortest route as Distance $(v)=(I B Z 1+I C Z 1+I C Y 1+I C X 1+I B X 1=5+$ $2.75+1.75+2=11.5 \mathrm{~km}$ ).

The proof of Dijkstra's algorithm is derived from the acceptance of the number of visited intersections in Table 1.

Table 1. Calculating process.

\begin{tabular}{cccccccccc}
\hline & IBZ1 & IAZ1 & ICZ1 & IAY1 & IBY1 & ICY1 & ICX1 & IAX1 & IBX1 \\
\hline v = & 0 & $\infty$ & $\infty$ & $\infty$ & $\infty$ & $\infty$ & $\infty$ & $\infty$ & $\infty$ \\
v = 1:IBZ1 & - & 2.5 & 5 & $\infty$ & 7.5 & $\infty$ & $\infty$ & $\infty$ & $\infty$ \\
v = 2:IAZ1 & - & - & 5 & 6 & 7.5 & $\infty$ & $\infty$ & $\infty$ & $\infty$ \\
v = 3:ICZ1 & - & - & - & 6 & 7.5 & 7.75 & $\infty$ & $\infty$ & $\infty$ \\
v = 4:IAY1 & - & - & - & - & 7.5 & 7.75 & $\infty$ & 11 & 13 \\
v = 5:IBY1 & - & - & - & - & - & 7.75 & $\infty$ & 11 & 13 \\
v = 6:ICY1 & - & - & - & - & - & - & 9.5 & 11 & 13 \\
v = 7:ICX1 & - & - & - & - & - & - & - & 11 & 11.5 \\
v = 8:IAX1 & - & - & - & - & - & - & - & - & 11.5 \\
w & 0 & 2.5 & 5 & 6 & 7.5 & 7.75 & 9.5 & 11 & 11.5 \\
\hline
\end{tabular}




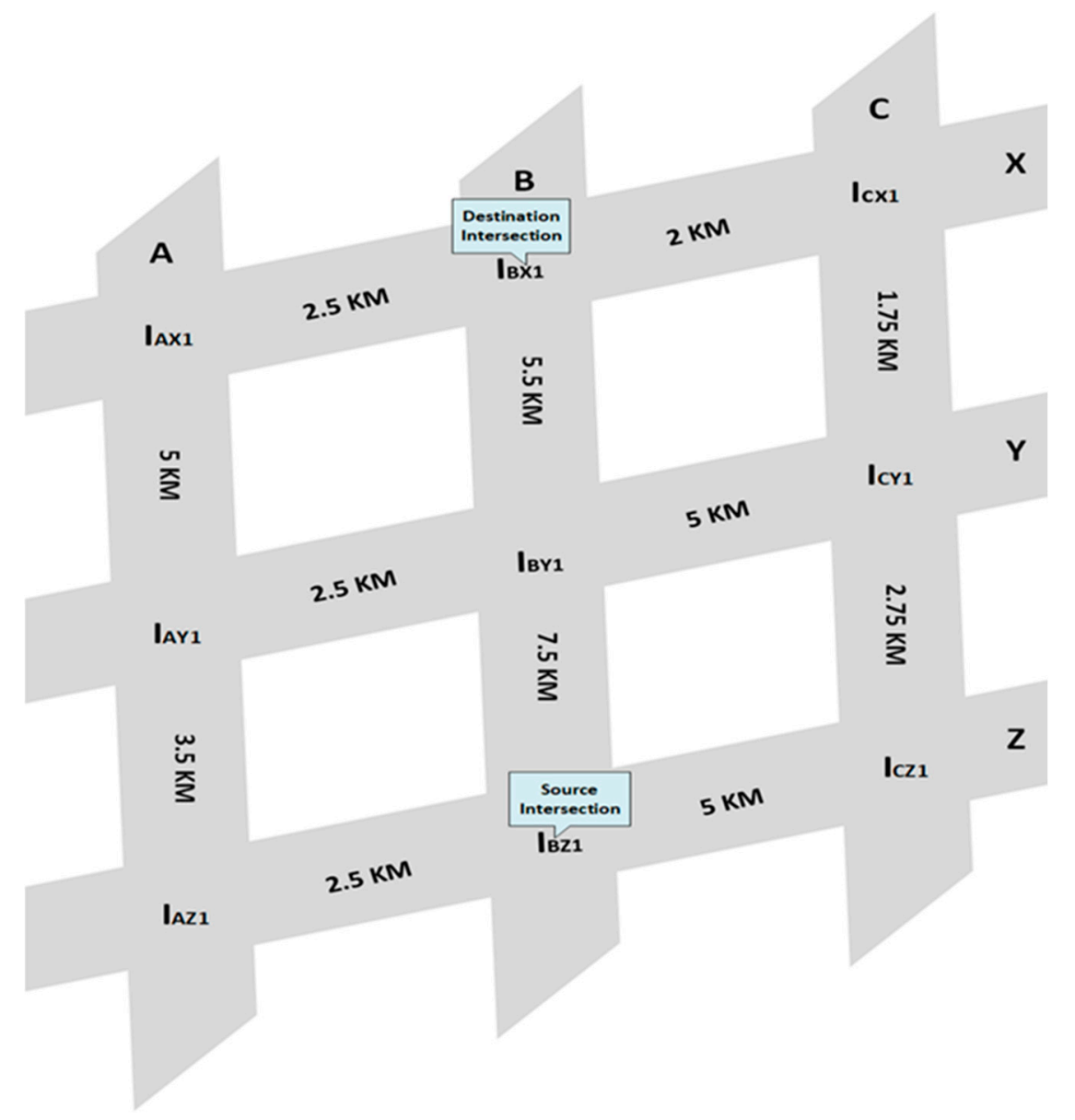

Figure 3. Shortest route discovery metric.

Assumption: For each intersection $v$, when traveling only through the visited intersection, source to $\mathrm{v}$, distance $(\mathrm{v})$ is the shortest route; if the source to $\mathrm{v}$ route does not exist, it is infinite $(\infty)$. (Note: We do not assume that distance (v) is the actual shortest route for an unknown intersection.) The basic situation is that there is only one visited intersection, the first intersection, in this case, the assumption is negligible. Otherwise, assume the assumption of n- 1 access intersections. For example, we select $(\mathrm{v}, \mathrm{u})$ as an edge, where $\mathrm{u}$ has the minimum distance $(\mathrm{u})$ of any undefined intersection, and the edge $(v, u)$ makes distance $(\mathrm{u})=$ distance $(v)+$ length $(\mathrm{v}, \mathrm{u})$. The distance $(\mathrm{u})$ is considered to be the shortest route from the source to $u$, because if there is a shorter route, and if $w$ is the first undefined intersection on the route, then according to the original assumption, the distance $(w)>$ distance $(u)$ causes incompatibility. Similarly, if there is a shorter route to $u$ without using an undefined intersection, and if the last intersection on that route is $\mathrm{w}$, then we will get distance $(\mathrm{u})=$ distance $(\mathrm{w})+$ length $(\mathrm{w}$, $\mathrm{u}$ ), also incompatible. After processing with $\mathrm{u}$, it will still be true that for each undefined intersection $\mathrm{w}$, distance $(\mathrm{w})$ will be the shortest route from the source to $\mathrm{w}$ using the visited intersection. If there is a short path that does not go through $u$, we have already found it, and if there is a short path using $u$, we will update it during $u$ processing.

After the intersection is visited, the shortest route from source to any intersection $\mathrm{v}$ consists only of the visited intersection. Therefore distance $(\mathrm{v})$ is the shortest route.

Balanced to Avoid Potential Congestion: In our model, we are using a simple trick to regulate congestion before it occurs, by previous perception data we can say the maximum probability of congestion period is in the morning and in the afternoon. Basically, on holidays, during this time, the control room takes appropriate action by using UAVs to regulate congestion. We consider our RSUs maximum capacity of fifty nodes at a time in an intersection. If more than fifty nodes at a time appear is an intersection, then the RSU cannot control it. Packet loss will be started before starting 
this predicament. The control room will take action and quickly launch UAVs to increase the RSUs' coverage and regulate potential congestion in this intersection.

\section{Experiments}

In this section, we analyze the performance of our proposed UAV-assisted (UAVa) protocol to study the efficiency of UAVa and compare its overall performance with the traditional routing protocol AODV. We considered the realistic urban scenario in Dhaka, Bangladesh, as shown in Figure 4a-c. Figure 4a represents the SUMO software view of our simulated area, Figure $4 \mathrm{~b}$ represents the OpenStreetMap view of our simulated area, and Figure $4 \mathrm{c}$ represents the actual geographical view of our simulated area. We used the simulation tool Network Simulator (NS-2.35) to evaluate UAVa [28]. Using two mobility generator tools, Simulation of Urban Mobility (SUMO) [29], and VanetMobiSim [30], to generate realistic movements for vehicles and UAVs, respectively, the tools know the flexibility and sustainability of nodes movement. The hybrid random walk mobility model for UAVs describes the UAV movement which may cover congested intersection areas. Geographic data were imported from OpenStreetMap (OSM) to integrate the road layout into our simulation [30].

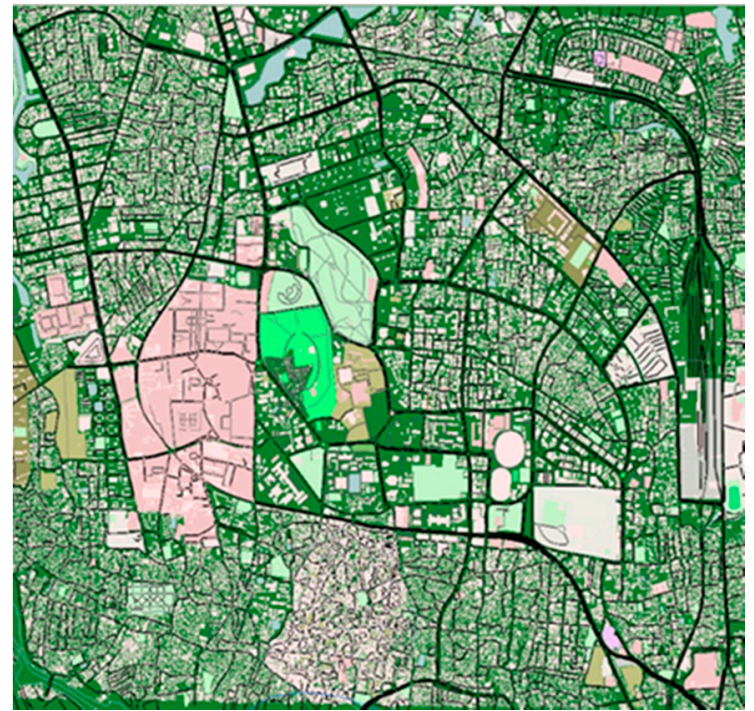

(a)

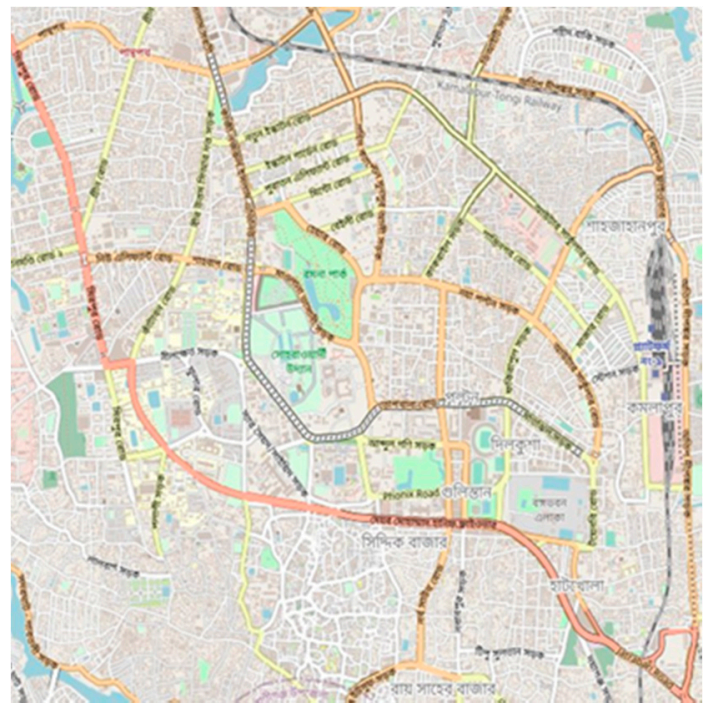

(b)

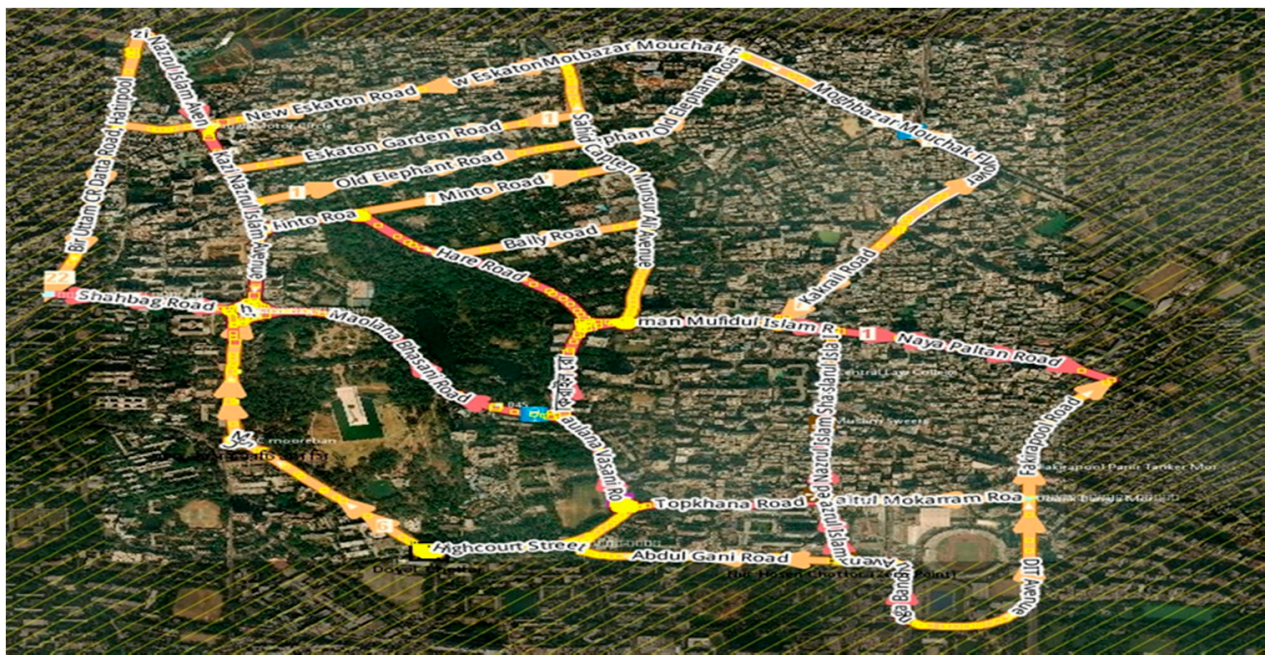

(c)

Figure 4. Map of the simulated area in Dhaka, Bangladesh: (a) SUMO software view of our simulated area. (b) OpenStreetMap view of our simulated area. (c) actual geographical view of our simulated area. 
A. Simulation Setup:

The vehicle simulation was conducted in the $9000 \mathrm{~m} \times 4000 \mathrm{~m}$ area shown in Figure 4c. The initial position of the node is distributed randomly. This urban map is stretched, encompassing nearly 20 intersections and 46 road segments. The vehicles moved with speed not more than $40 \mathrm{~km} / \mathrm{h}$, and UAVs moved with speed not more than $60 \mathrm{~km} / \mathrm{h}$. We assumed that the coverage area of all RSUs did not surpass $200 \mathrm{~m}$, and UAVs did not surpass $300 \mathrm{~m}$.

To simulate this network, we used 106 vehicles, 15 RSUs, and 4 UAVs. The simulation parameters are given in Table 2. The communication range of the node is set to $200 \mathrm{~m}$, and the channel's data transmission rate is set to $6 \mathrm{Mbps}$. We used the IEEE $802.11 \mathrm{p}$ specification for implementation of the MAC layer. For each node pair (source-destination), and packet size (512 bytes), we found the data from Constant Bit Rate (CBR). We also adopted User Datagram Protocol (UDP) and Transmission Control Protocol (TCP) as transportation layer protocols for our project, UDP for RSU to Vehicle, UAV to Vehicle, and RSU to UAV communications and TCP for internal communication between cellular networks.

Table 2. Simulation parameters.

\begin{tabular}{ccc}
\hline Parameter & Value & Unit \\
\hline Simulation Area & $9000 \times 4000$ & $\mathrm{~m}^{2}$ \\
Road Intersection Number & 20 & - \\
Road Segments Number & 46 & - \\
Vehicular Speed Limits & $0-40$ & $\mathrm{~km} / \mathrm{h}$ \\
UAV Speed Limits & $0-60$ & $\mathrm{Km} / \mathrm{h}$ \\
RSUs Number & 15 & - \\
RSUs Transmission Range & 200 & $\mathrm{~m}$ \\
Vehicle Number & $0-106$ & - \\
UAV Number & $0-4$ & - \\
UAVs Transmission Range & 300 & $\mathrm{~m}$ \\
Transport Layer & TCP, UDP & - \\
Channel Capacity & 6 & $\mathrm{Mbps}$ \\
Packet Size & 512 & bytes \\
Packet Type & CBR & - \\
Node Energy & 300 & joule \\
Simulation Run Time & 180 & $\mathrm{~s}$ \\
\hline
\end{tabular}

In our project, we are using UAVs as a relay on an emergency basis to make our simulations more accurate. Hello packets are broadcasted every 1 (s). It is worth mentioning that the transmission range of vehicles and RSUs are considered the same when communicating between them $(\approx 200 \mathrm{~m})$, and the transmission ranges of nodes and UAVs in congested intersections will be considered the same when the same communication is established $(\approx 300 \mathrm{~m})$.

Based on multiple tests in the simulation environment, we can say that the chances of success in radio transmission between RSU and UAV are great. The probability of success is too high for hybrid radio transmission (between RSUs and cellular networks). In order to evaluate the simulation protocol more realistically, the initial geographic location of the node and RSU has been well allocated through the network. UAVs and nodes' mobility is regulated, as their movement is restricted only in the simulated area. Every simulation has a total time set to $180 \mathrm{~s}$.

The other simulation parameters are summarized in Table 2. Certain parameters were chosen based on previous research [8,31-33]. In this way, based on multiple metrics, some evaluation indicators are estimated, such as the average throughput, the instant throughput, the packet delivery ratio, the residual energy, and the end-to-end delay. 
Average throughput: Represents the average rate of successfully receiving packets $R p$ over a communication channel from stop time Tsp to start time Ts.

$$
\text { Average throughput }=\frac{R p}{\mathrm{Tsp}-\mathrm{Ts}} \times \frac{8}{100}(\text { in kbps })
$$

Instant throughput: Represents the packets received successfully at the current time and packet size R (Receive packets $R p$ + Receive packet size Rz) over a communication channel, where time is $\mathrm{T}$, current time Tcs, and previous Time Tps.

$$
\text { Instant throughput }=\frac{\mathrm{R}}{\mathrm{Tcs}+(\mathrm{T}-\mathrm{Tps})} \times \frac{8}{100}(\text { in } \mathrm{kbps})
$$

Packet Delivery Ratio (PDR): Represents the fraction of the data packets delivered from the source nodes to the destination nodes over a communication channel, where $R p$ is the receive packets, and $R s$ is the sent packets $n$ represents the number of sensor nodes.

$$
\text { Packet Delivery Ratio }(P D R)=\frac{R p}{\sum_{\mathrm{i}=1}^{\mathrm{n}} R s} \times 100
$$

Residual Energy: Represents the remaining node energy, where $E$ is the total node energy and overall energy consumption $E c$ by a node.

$$
\text { Residual energy }=E-E c \text { (in joule) }
$$

End-to-End Delay: Represents the average value of all successfully received packets delay $D n$ and receive packet $R p$, the total number of nodes $n$.

$$
\text { End - to-End delay }=\frac{\sum_{\mathrm{n}=1}^{R p} D n}{R p}
$$

B. Experimental Result Analysis:

In this sub-section we will present our experimental results and its analysis.

i. Average Throughput:

The average throughput is shown in Figure 5, with traditional AODV and our proposed UAVa protocol. For both UAVa and AODV protocols, start time is $0 \mathrm{~s}$, stop time is $179 \mathrm{~s}$, UAVa's received packets are 95,331, and AODV's received packets are 90,148, average throughput for UAVa is $4.237 \mathrm{kbps}$, and for AODV is $4.010 \mathrm{kbps}$. The UAVa performed better than the AODV, which is reasonably justified. 


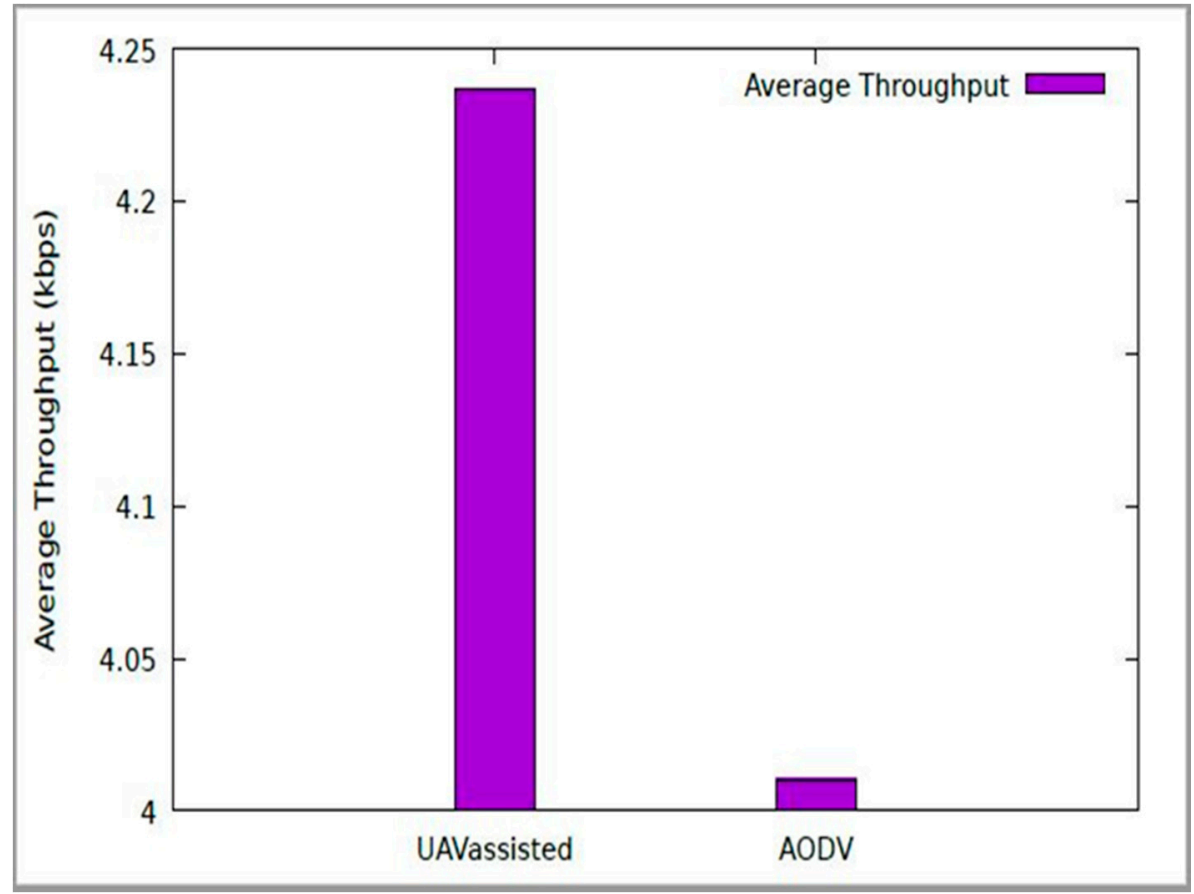

Figure 5. Average throughput.

ii. Instant Throughput:

Figure 6 compares instant throughput with a different time periods of simulations for UAVa and AODV protocols. We can consider along with the different time periods, the instant throughput is changed, and for AODV protocol it is changed rapidly, but for the UAVa protocol, it is changed a bit, so we can say that UAVa protocol has performed better than AODV protocol for instant throughput measured in kbps for each case.

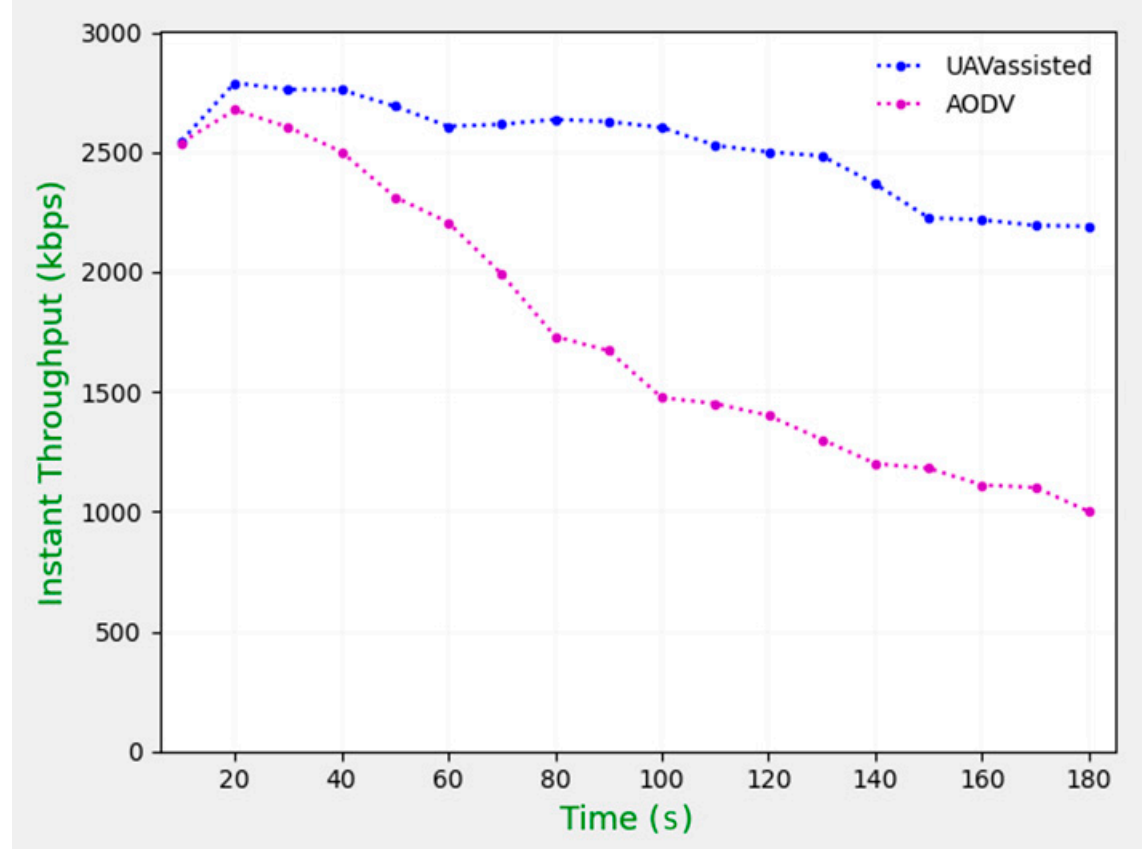

Figure 6. Instant throughput. 
iii. $\quad$ Packet Delivery Ratio (PDR):

Figure 7 shows the variation of PDR for the UAVa and AODV protocols. Here, we can consider according to the increasing number of nodes that packet delivery ratio is increased for both AODV and UAVa protocols, but for UAVa protocols packet delivery ratio is better than AODV. The highest PDR is achieved for UAVa, again proving its effectiveness. It depends on both vehicles and RSUs or UAVs (both RSUs and UAVs can carry packets), which can be checked at all times in the data transmission.

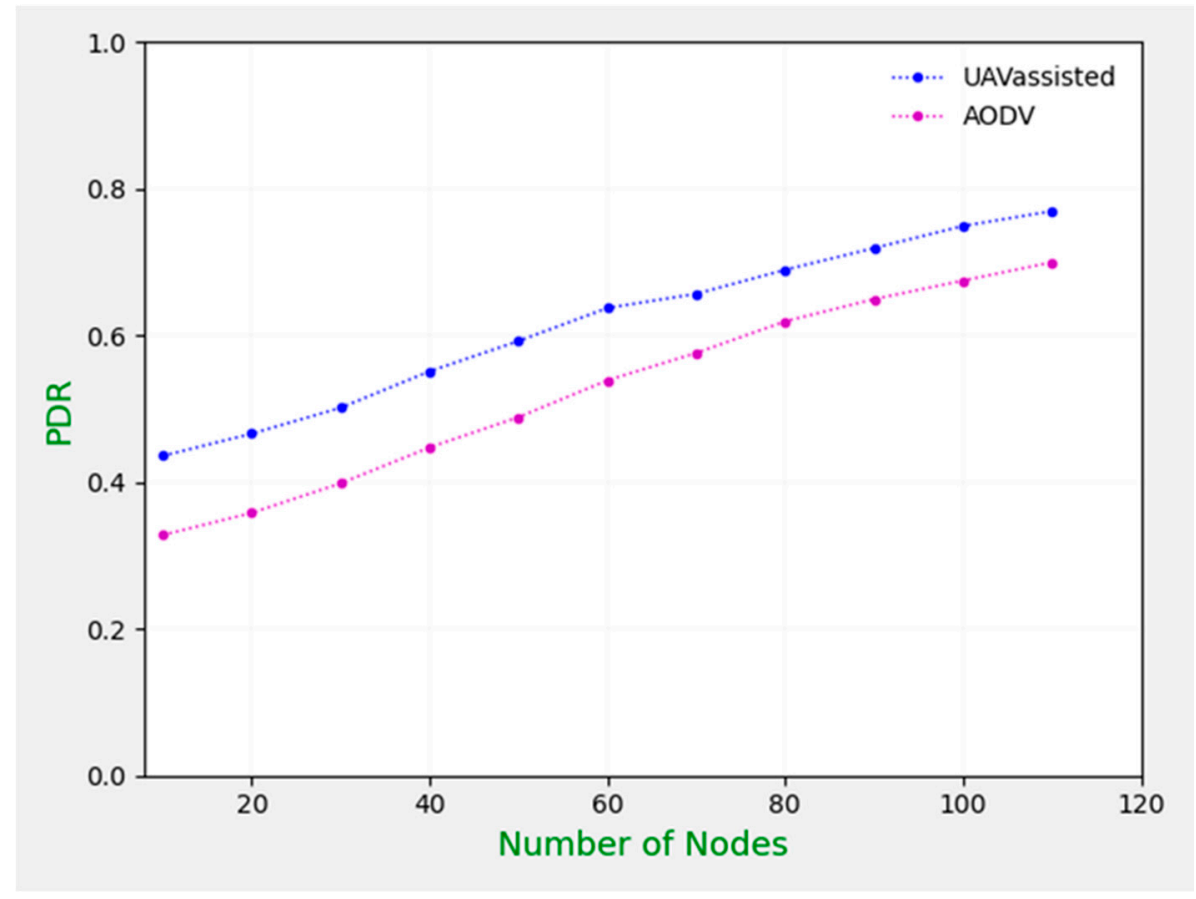

Figure 7. Packet delivery ratio.

iv. Residual Energy:

Figure 8 shows the residual energy under a varying number of nodes for our proposed UAVa and AODV protocols. We put initial energy as 300 joules for all nodes, transmission power $0.9 \mathrm{~W}$ and receiving power $0.7 \mathrm{~W}$, ideal power $0.45 \mathrm{~W}$, sleep power $0.05 \mathrm{~W}$, transition power $0.02 \mathrm{~W}$, and transition time $0.005 \mathrm{~s}$ for both UAVa and AODV protocols. After simulation run times, we can calculate remaining energy for all nodes, and it is good news that our proposed UAVa protocol works better than the existing AODV protocol. For UAVa protocol, the remaining energy level is better than the AODV protocol. We can decide that our proposed protocols remaining energy is much better than the AODV protocol. We can call it a new energy saving model for VANETs. 


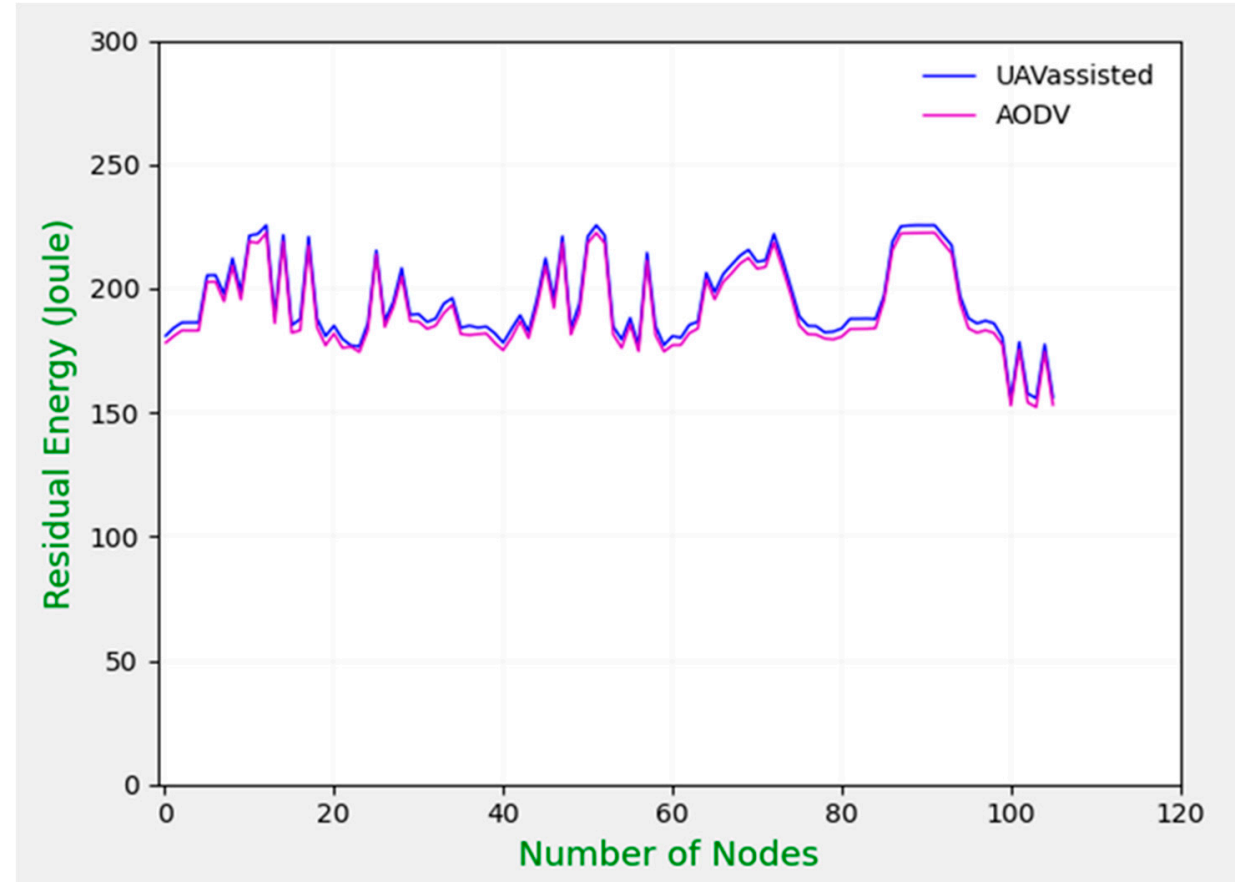

Figure 8. Residual energy.

v. End-to-End Delay:

Figure 9 provides a contrast with static CBR connections with various numbers of nodes and end-to-end delays. In general, as the number of nodes increases, the end-to-end delays decrease for both UAVa and AODV, whereas increasing the number of nodes means increasing the network bandwidth, leading to a reduction in the maximum local situation. Due to the use of RSUs and UAVs, the performance of UAVa is better than AODV.

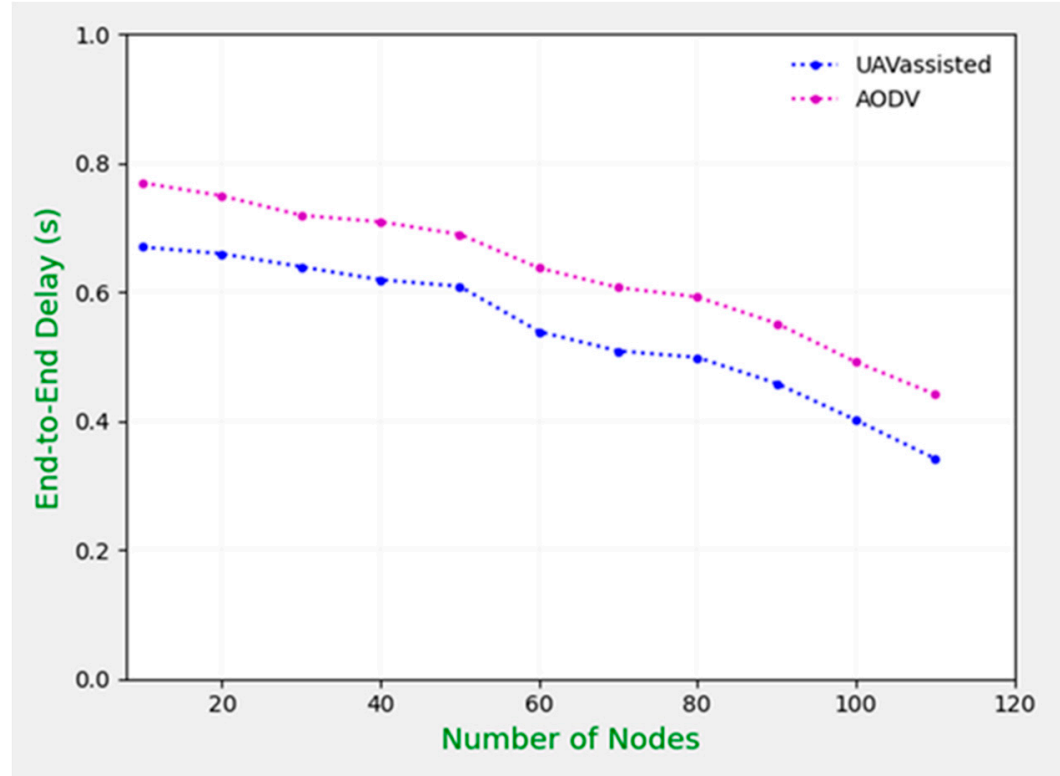

Figure 9. End-to-end delay. 


\section{Conclusions}

In VANET, nodes travel quickly, and the topology also changes, which is why routing architecture for VANET is very difficult. In this article, we have done an in-depth survey on AODV, which is more appropriate in VANET. The UAVa protocol and its extensions have been implemented to support ad hoc routing within RSUs, UAVs, and VANETs, as well as RSUs themselves. The UAVa protocol is dedicated to urban vehicle environments, and its primary goal is to enhance the performance of routing protocols based on intersections. UAVs are used to assist RSUs when congestions occur in intersections. When vehicle density is too high at intersections, RSUs are also used to route data packets via UAVs. We performed a simulation-based performance assessment of our UAVa protocols and demonstrated the efficacy and robustness of UAVa in terms of adding routing efficiency, average throughput, instant throughput, reduced latency, and improved delivery ratio and residual energy compared to AODV protocols.

In our future work, we will try to reduce the number of RSUs, and save more energy. We intend to apply UAV-assisted along with table-driven routing protocols. A comparative study can also be made based on our proposed work with table-driven routing protocols. UAV-assisted protocols will be strengthened in terms of protection and adapted to other areas such as rural scenarios and highways.

Author Contributions: Conceptualization, S.J., Y.Z., and M.A.I.H.; data curation, S.J.; formal analysis, Y.Z. and F.A.; investigation, Y.Z.; methodology, S.J.; supervision, Y.Z.; validation, M.A.I.H.; visualization, S.J., M.A.I.H., and F.A.; writing—original draft, S.J. All authors have read and agreed to the published version of the manuscript.

Funding: This research was funded by [the Fundamental Research Funding for the Central Universities of Ministry of Education of China] grant number [18D110408], [the Special Project Funding for the Shanghai Municipal Commission of Economy and Information Civil-Military Inosculation Project “Big Data Management System of UAVs"] grant number [JMRH-2018-1042], and [the National Natural Science Foundation of China (NSFC)] grant number [18K10454]. The APC was funded by [the Fundamental Research Funding for the Central Universities of Ministry of Education of China].

Conflicts of Interest: The authors declare no conflict of interest.

\section{References}

1. Liu, X.; Jaekel, A. Congestion control in V2V safety communication: Problem, analysis, approaches. Electronics 2019, 8, 540. [CrossRef]

2. Spadaccino, P.; Cuomo, F.; Baiocchi, A. Epidemic and timer-based message dissemination in VANETs: A performance comparison. Electronics 2020, 9, 595. [CrossRef]

3. Wang, J.; Shao, Y.; Ge, Y.; Yu, R. A survey of vehicle to everything (V2X) testing. Sensors 2019, $19,334$. [CrossRef]

4. Vidya, V.M.; Deepa, N. Big data analytics in intelligent transportation systems using hadoop. Int. J. Recent Technol. Eng. 2019, 7, 75-80.

5. Papadimitratos, P.; La Fortelle, A.; Evenssen, K.; Brignolo, R.; Cosenza, S. Vehicular communication systems: Enabling technologies, applications, and future outlook on intelligent transportation. IEEE Commun. Mag. 2009, 47, 84-95. [CrossRef]

6. Guo, C.; Li, D.; Zhang, G.; Zhai, M. Real-time path planning in urban area via VANET-Assisted traffic information sharing. IEEE Trans. Veh. Technol. 2018, 67, 5635-5649. [CrossRef]

7. Aouiz, A.A.; Boukli Hacene, S.; Lorenz, P.; Gilg, M. Network life time maximization of the AOMDV protocol using nodes energy variation. Netw. Protoc. Algorithms 2018, 10, 73. [CrossRef]

8. Sam, D.; Velanganni, C.; Evangelin, T.E. A vehicle control system using a time synchronized Hybrid VANET to reduce road accidents caused by human error. Veh. Commun. 2016, 6, 17-28. [CrossRef]

9. Khan, U.A.; Lee, S.S. Multi-layer problems and solutions in VANETs: A review. Electronics 2019, 8, 204. [CrossRef]

10. Amruta, M. Efficient routing protocol in MANET. Int. J. Recent Innov. Trends Comput. Commun. 2015, 3, 2234-2238. [CrossRef]

11. Malik, S.; Sahu, P.K. A comparative study on routing protocols for VANETs. Heliyon 2019, 5, e02340. [CrossRef] [PubMed] 
12. Pradeep Kumar, T.S.; Chitnis, S. Distance and hops-based energy estimation in wireless sensor networks. Asian J. Pharm. Clin. Res. 2017, 10, 85-89. [CrossRef]

13. Shrestha, R.; Bajracharya, R.; Nam, S.Y. Challenges of future VANET and cloud-based approaches. Wirel. Commun. Mob. Comput. 2018, 2018, 5603518. [CrossRef]

14. Santa, J.; Fernández, P.J.; Ortiz, J.; Sanchez-Iborra, R.; Skarmeta, A.F. SURROGATES: Virtual OBUs to foster 5G vehicular services. Electronics 2019, 8, 117. [CrossRef]

15. Hamid, B.; El Mokhtar, E.N. Performance analysis of the Vehicular Ad hoc Networks (VANET) routing protocols AODV, DSDV and OLSR. In Proceedings of the 5th International Conference on Information and Communication Technology and Accessibility, ICTA 2015, Marrakesh, Morocco, 21-23 December 2015. [CrossRef]

16. Kumar, P.; Kataria, H.S.; Ghosh, T. Congestion control approach by reducing the number of messages in VANET. In Proceedings of the 4th International Conference on Reliability, Infocom Technologies and Optimization: Trends and Future Directions, ICRITO 2015, Noida, India, 2-4 September 2015; pp. 7-11. [CrossRef]

17. Zeadally, S.; Hunt, R.; Chen, Y.S.; Irwin, A.; Hassan, A. Vehicular ad hoc networks (VANETS): Status, results, and challenges. Telecommun. Syst. 2012, 50, 217-241. [CrossRef]

18. Taleb, M.Y.; Merniz, S.; Harous, S. Congestion control techniques in VANETs: A survey. In Proceedings of the 13th International Wireless Communications and Mobile Computing Conference, IWCMC 2017, Valencia, Spain, 26-30 June 2017; pp. 484-488. [CrossRef]

19. Nzouonta, J.; Rajgure, N.; Wang, G.; Borcea, C. VANET routing on city roads using real-time vehicular traffic information. IEEE Trans. Veh. Technol. 2009, 58, 3609-3626. [CrossRef]

20. Zahedi, K.; Zahedi, Y.; Ismail, A.S. CJBR: Connected junction-based routing protocol for city scenarios of VANETs. Telecommun. Syst. 2019, 72, 567-578. [CrossRef]

21. Sami Oubbati, O.; Chaib, N.; Lakas, A.; Bitam, S.; Lorenz, P. U2RV: UAV-assisted reactive routing protocol for VANETs. Int. J. Commun. Syst. 2020, 33, e4104. [CrossRef]

22. Silva, A.; Reza, N.; Oliveira, A. Improvement and performance evaluation of GPSR-based routing techniques for vehicular Ad Hoc networks. IEEE Access 2019, 7, 21722-21733. [CrossRef]

23. Guo, C.; Li, D.; Zhang, G.; Ding, X.; Curtmola, R.; Borcea, C. Dynamic interior point method for vehicular traffic optimization. IEEE Trans. Veh. Technol. 2020, 69, 4855-4868. [CrossRef]

24. Xu, M.; Li, D.; Zhang, G.; Cao, M.; Liao, S. A path planning approach with maximum traffic flow and minimum breakdown probability in complex road network. In Lecture Notes in Computer Science (Including Subseries Lecture Notes in Artificial Intelligence and Lecture Notes in Bioinformatics); Springer: Shanghai, China, 2018; Volume 11280 LNCS, pp. 92-102. [CrossRef]

25. Azari, M.M.; Geraci, G.; Garcia-Rodriguez, A.; Pollin, S. Cellular UAV-to-UAV Communications. In Proceedings of the IEEE International Symposium on Personal, Indoor and Mobile Radio Communications, PIMRC 2019, Istanbul, Turkey, 8-11 September 2019. [CrossRef]

26. Sailaja, P.; Ravi, B.; Jaisingh, T. Performance Analysis of AODV and EDAODV Routing Protocol under Congestion Control in VANETs. In Proceedings of the International Conference on Inventive Communication and Computational Technologies, ICICCT 2018, Coimbatore, India, 20-21 April 2018; pp. 945-948. [CrossRef]

27. Oubbati, O.S.; Lakas, A.; Zhou, F.; Güneş, M.; Lagraa, N.; Yagoubi, M.B. Intelligent UAV-assisted routing protocol for urban VANETs. Comput. Commun. 2017, 107, 93-111. [CrossRef]

28. Shin, J.Y.; Jang, J.W.; Kim, J.M. Result based on ns2, simulation and emulation verification. In Proceedings of the 2009 International Conference on New Trends in Information and Service Science, NISS 2009, Beijing, China, 30 June-2 July 2009; pp. 807-811. [CrossRef]

29. Lim, K.G.; Lee, C.H.; Chin, R.K.Y.; Beng Yeo, K.; Teo, K.T.K. SUMO enhancement for vehicular ad hoc network (VANET) simulation. In Proceedings of the 2017 IEEE 2nd International Conference on Automatic Control and Intelligent Systems, I2CACIS 2017, Sabah, Malaysia, 21 October 2017; pp. 86-91. [CrossRef]

30. Mousavi, S.M.; Rabiee, H.R.; Moshref, M.; Dabirmoghaddam, A. MobiSim: A framework for simulation of mobility models in mobile ad-hoc networks. In Proceedings of the 3rd IEEE International Conference on Wireless and Mobile Computing, Networking and Communications, WiMob 2007, White Plains, NY, USA, 8-10 October 2007. [CrossRef]

31. Haider, S.; Abbas, Z.H.; Abbas, G.; Waqas, M. A Novel Cross-Layer V2V Architecture for Direction-Aware Cooperative Collision Avoidance. Electronics 2020, 9, 1112. [CrossRef] 
32. Ahsan, W.; Khan, M.F.; Aadil, F.; Maqsood, M.; Ashraf, S.; Nam, Y.; Rho, S. Optimized node clustering in VANETs by using meta-heuristic algorithms. Electronics 2020, 9, 394. [CrossRef]

33. Wu, Q.; Xia, S.; Fan, Q.; Li, Z. Performance analysis of IEEE 802.11p for continuous backoff freezing in IoV. Electronics 2019, 8, 1404. [CrossRef] 